\title{
Measuring health-related quality of life in children with cancer living in mainland China: feasibility, reliability and validity of the Chinese mandarin version of PedsQL 4.0 Generic Core Scales and 3.0 Cancer Module
}

\author{
Yi Ji', Siyuan Chen ${ }^{2}$, Kai Li ${ }^{1}$, Nong Xiao ${ }^{3}, X^{\prime}$ Yue Yang ${ }^{3}$, Shan Zheng ${ }^{1}$ and Xianmin Xiao ${ }^{1 *}$
}

\begin{abstract}
Background: The Pediatric Quality of Life Inventory (PedsQL) is widely used instrument to measure pediatric health-related quality of life (HRQOL) for children aged 2 to 18 years. The purpose of the current study was to investigate the feasibility, reliability and validity of the Chinese mandarin version of the PedsQL 4.0 Generic Core Scales and 3.0 Cancer Module in a group of Chinese children with cancer.

Methods: The PedsQL 4.0 Genetic Core Scales and the PedsQL 3.0 Cancer Module were administered to children with cancer (aged 5-18 years) and parents of such children (aged 2-18 years). For comparison, a survey on a demographically group-matched sample of the general population with children (aged 5-18) and parents of children (aged 2-18 years) was conducted with the PedsQL 4.0 Genetic Core Scales.

Result: The minimal mean percentage of missing item responses (except the School Functioning scale) supported the feasibility of the PedsQL 4.0 Generic Core Scales and 3.0 Cancer Module for Chinese children with cancer. Most of the scales showed satisfactory reliability with Cronbach's $\alpha$ of exceeding 0.70 , and all scales demonstrated sufficient test-retest reliability. Assessing the clinical validity of the questionnaires, statistically significant difference was found between healthy children and children with cancer, and between children on-treatment versus offtreatment $\geq 12$ months. Positive significant correlations were observed between the scores of the PedsQL 4.0 Generic Core Scale and the PedsQL 3.0 Cancer Module. Exploratory factor analysis demonstrated sufficient factorial validity. Moderate to good agreement was found between child self- and parent proxy-reports.
\end{abstract}

Conclusion: The findings support the feasibility, reliability and validity of the Chinese Mandarin version of PedsQL 4.0 Generic Core Scales and 3.0 Cancer Module in children with cancer living in mainland China.

\section{Background}

Currently, an increasing number of children with cancer are cured for their diseases in the world, including China. However, common treatments such as surgery, radiotherapy, chemotherapy, whether palliative or curative, can have deleterious side-effects affecting many aspect of the quality of children's lives - not only

\footnotetext{
* Correspondence: xmxiao@shmu.edu.cn

${ }^{1}$ Department of Pediatric Surgery, Children's Hospital of Fudan University, Shanghai, 201102, China

Full list of author information is available at the end of the article
}

physical aspects but also social and emotional aspects $[1,2]$. Survivors may experience a number of long-term adverse effects from the tumor and its treatment [3-6]. Focusing on the patients' psychosocial and social wellbeing in addition to their physical health is an essential requirement in accordance with the WHO definition of health and well-being [7].

Health-related quality of life (HRQOL) is a continuous concept influenced by a person's objective assessments of function or health status as well as subjective perceptions of their personal health [8]. It is increasingly 
acknowledged as an important health outcome measure in clinical trials and health services research and evaluation. However, one challenge in measuring HRQOL in children is that the instrument must account for the continuous physical, emotional, social and cognitive development during childhood and adolescence. Another problem is the questionnaires should provide the required information [9]. Although there are a number of generic HRQOL instruments applicable to children, assessment has relied exclusively on proxy-report [10], or the concordance between self- and proxy-report has been demonstrated to be imperfect [11]. Given the multidimensional impact cancer has on children, it is necessary to find an appropriate instrument to capture and evaluate the HRQOL of these children.

The Pediatric Quality of Life Inventory (PedsQL) is one of the more promising HRQOL measures for children aged 2-18 years $[12,13]$. The advantages of PedsQL included brevity, availability of age-appropriate version, and parallel for child and parent. The approach is modular, with Generic Core Scales complemented by disease-specific modules, such as the PedsQL Cancer Module [14-19]. The PedsQL 4.0 Generic Core Scales was specifically designed for application in both healthy and patient populations. The PedsQL 3.0 Cancer Module was designed to measure HRQOL dimensions specific to pediatric cancers. Studies with PedsQL indicate that both healthy children and children with cancer aged 5-18 years can self-report their HRQOL [20-22].

As the population of Chinese children nears 300 million, a conservative projection of 45 thousands new cases of pediatric cancer each year can be made [23]. However, limited information is available to understand the HRQOL of children with cancer living in mainland China. The objective of this study was to investigate the feasibility, reliability, and validity of the Chinese mandarin version of the PedsQL 4.0 Generic Core Scales and 3.0 Cancer Module in children with cancer.

\section{Method}

\section{Participants and Settings}

This study was developed in the cities of Shanghai and Chongqing, China. We recruited children with cancer and their parents by means of convenience samples from 2 children's hospitals at the cities. Children aged 5 to 18 years who were diagnosed with cancer were included in this study, and the parents were included if their child was 2 to 18 years old. Children were excluded from this study if they had comorbid disease or major developmental disorders. The sample size needed to procedure medium correlation (0.30) in the examination of discriminant validity was calculated. 154 subjects were needed to take part in the study when we set the type I error at $1 \%$ and the statistical power at
$90 \%$. We estimated that approximately more than 60$70 \%$ of participants would agree to participate. Finally, two hundred and seventy five families were approached for the study. Controls were 300 families of healthy children aged 2 to 18 years recruited from schools, with distributional matching to the patients on age and gender.

\section{Measurement}

\section{PedsQL 4.0 Generic Core Scales}

The 23-item PedsQL 4.0 Generic Core Scales encompasses the essential core domains for pediatric HRQOL measurement: (1) physical functioning (8 items), (2) emotional functioning (5 items), (3) social functioning (5 items), and (4) school functioning (5 items). It comprises of parallel child self-report and parent proxyreport format. Child self-report includes ages 5-7 (young children), 8-12 (children), and 13-18 (teens) years. Patent proxy-report includes ages 2-4 (toddlers), 5-7, 812, and 13-18 years. The items for each of the forms are essentially identical, differing in developmentally appropriate language, or first or third person tense. The instructions ask how much of a problem each item has been during the past one month. A 5-point response scale is utilized across child self-report for ages 8-18 and parent proxy-report $(0=$ never a problem; $1=$ almost never a problem; 2 = sometimes a problem; 3 = often a problem; $4=$ almost always a problem). The child-report for children aged 5-7 is completed with the help of an interviewer. To increase its ease of use, the rating scale has been reworded and simplified to a 3 point scale $(0=$ not at all a problem; $2=$ sometimes a problem; 4 = a lot of a problem), with each response choice anchored to a happy to sad faces scale. Items are reverse-scored and linearly transformed to a 0-100 scale, with higher scores indicating a better HRQOL. Scale scores are computed as the sum of the items divided by the number of items answered. If more than $50 \%$ of the items in the scale are missing, the scale score is not computed. To create the Psychosocial Functioning score, the mean is computed as the sum of the items divided by the number of the items answered in the Emotional, Social, and School Functioning Scale.

\section{PedsQL 3.0 Cancer Module}

The PedsQL 3.0 Cancer Module instrument encompasses 8 subscales: (1) pain and hurt (2 items), (2) nausea (5 items), (3) procedural anxiety (3 items), (4) treatment anxiety (3 items), (5) worry (3 items), (6) cognitive problems (5 items), (7) perceived physical appearance (3 items), and (8) communication (3 items). The cognitive problems scales were modified to include fewer items for children aged 2-7 years. The format, instructions, Likert response scale, and scoring method of the Cancer Module are identical to the PedsQL 4.0 Generic Core Scales [24]. 


\section{Procedure}

The investigation was performed by 4 research students majoring in Pediatrics and 2 nurses. All of them were trained in interviewing and administering questionnaires. On-treatment status was defined as patients who were receiving medical treatment to induce remission. Off-treatment status was defined as patients who completed all therapy by the time of the assessment $[25,26]$. The on-treatment patient was interviewed while hospitalized. The off-treatment patient was interviewed during clinic visits. Parents were interviewed first, and were asked whether they would allow their children to participate in the study. The parents and their children completed the questionnaire independently during the pediatric patients' hospitalization or outpatient department visit. All the parents were asked to fill out the PedsQL 4.0 Generic Core Scales and the Cancer Module. The children were required to complete the questionnaires by self-administration except the Young Children by interview-administration [19]. The interviewers were available to assist the completion of the questionnaires if the parents/children had questions on semantic or conceptual understanding. They were also responsible for collecting and checking the questionnaires to ensure that there were no missing data or logical mistakes. Test-retest reliability was assessed at Children's Hospital of Fudan University (the same hospital in Shanghai). Thirty-four families with patients in stable condition according to their physician completed the PedsQL measures a second time (with an interval of 1-3 weeks between applications). For control group, the questionnaires interviews were conducted at schools. Written information was sent to parents who completed questionnaires at home, returning them to school by a specified date.

\section{Cross-culture adaptation and Ethical considerations}

The Chinese Mandarin Version was provided by MAPI Research Trust that translated the version according to their standardized procedures. Briefly, the instruments were translated independently into Chinese by two professional translators (native Chinese speakers) and translated back into English by two English native speakers (bilingual in Chinese). Then, a comparison between the backward version and the original version was assessed in order to detect any misunderstandings or mistranslations in the intermediary forward version of the questionnaires [27,28]. 15 children with cancer participated in pilot testing along with their parents. In consideration of the Chinese sociocultural environment, we avoided using the term 'cancer' or 'tumor' in questionnaires. The permission was obtained from its developer, James W. Varni, PhD. The human subject institutional review boards at each hospital approved the study. All subjects were given detailed written information about the methods, aims, and the voluntary nature of participation in the study, and written parental informed consent and child assent were obtained prior to enrollment.

\section{Statistical analysis}

Feasibility was determined from the average completion time and percentage of missing response. The average completion time was defined as the mean of completion time of the Generic Core Scales and Cancer Module. The percentage of all possible item-responses left unanswered was calculated for each subject on each single and summary scale and averaged over subjects [29]. The presence of floor and ceiling effects was assessed for the subscales scores and summary scores.

Scale internal consistency reliability was determined by calculating Cronbach's coefficient alpha. Scales with reliabilities of 0.70 or greater are recommended for comparing patients groups, while a reliability criterion of 0.90 is recommended for analyzing individual patient scale scores [30]. To determine retest reliability, the intra-class correlation coefficients (ICCs) between the initial test and retest scores was examined, with an ICC value of 0.40 representing moderate, 0.60 good, and 0.80 high agreement [31].

Discriminant validity was determined using the known-groups method. The Generic Core Scales scores were compared between groups differing in known health conditions (healthy children and children with cancer). HRQOL scores of children from the general population and children with cancer were compared using independent sample $t$ test. To determine the magnitude of the differences, effect sizes were evaluated. Effect size as utilized in these analyses was calculated by taking the difference between the healthy sample mean and the oncology sample mean, divided by the pooled standard deviation [32]. Additionally, analyses of variance also were conducted to examine whether there were differences in Generic Core Scales and Cancer Module scores among children with cancer on treatment and off-treatment ( $\leq 12$ months or $>12$ months). We hypothesized that healthy children would have higher PedsQL 4.0 scores (better HRQOL) than children with cancer. Moreover, we hypothesized that children with cancer on-treatment would report significant differences in HRQOL compared with those of children with cancer off-treatment based on previous studies $[19,33,34]$.

Construct validity for the Cancer Module was examined through an analysis between the Cancer Module Scales scores and relevant Generic Core Scales scores. It had been reported that computing the intercorrelations among scales provides initial information on the construct validity of an instrument. We hypothesized 
greater disease-specific symptoms or problems would correlate with lower overall generic HRQOL based on the conceptualization of disease-specific symptoms as causal indicators of generic HRQOL [35]. Correlation effect sizes were designed as small (0.01-0.29), medium (0.30-0.49), and large $(\geq 0.50)$. Pearson coefficients were utilized in these analyses.

Exploratory factor analysis was performed on the items to test the PedsQL underlying dimensions [36]. Principal component analysis with oblique rotation was performed to extract the factors. Factors with an eigenvalue less than 1.0 were disregarded.

Parent/child intercorrelations were computed to examine cross-informant variance [37]. Correlation effect sizes are designated as small (0.01-0.29), medium (0.30-0.49), and large ( $\geq 0.50)$.

Statistical analyses of the study were conducted by SPSS 16.0 for Window (SPSS, Inc, Chicago, USA) and the significance level was set at 0.05 .

\section{Results}

\section{Sample characteristics}

Of the patients group, 7 families refused to participate due to non-compliance, 2 children were later found to be ineligible and excluded from analysis, leaving 266 eligible families. The patients group was comprised of 202 children aged 5-18 years and 266 parents of children aged $2-18$ years. The mean age of the 154 boys (57.9\%) and 112 girls $(42.1 \%)$ was 8.8 years $(\mathrm{SD}=3.8)$. One hundred sixty-three patients (61.3\%) had been diagnosed with hematological diseases, and the remaining patients (38.7\%) had solid tumors. The mother answered the questionnaires in $82.7 \%$ of the cases and the father in $17.3 \%$ of the cases. 109 (41.0\%) patients completed all therapy by the time of assessment $(\mathrm{n}=109)$, and 74 (27.8\%) patients had been off treatment for over 12 months $(\mathrm{n}=74)$. Of the control group, 284 families returned the questionnaires, which gave a response rate of $94.7 \%$. The mean age of the 159 boys $(56.0 \%)$ and 125 girls $(44.0 \%)$ was 9.1 years $(\mathrm{SD}=3.9)$. Self-report forms are completed by 216 children (Table 1). There was no statistically significant difference between patients and healthy children refer to age and gender.

\section{Descriptive statistics}

As showed in Table 2 the Generic Core scores were consistently higher for child reports than for parent reports. No floor effects were seen in the patient group. We found ceiling effects both in child self- and parent proxy-reports ranging from $2.6 \%$ to $19.8 \%$ in the patient group and 5.6 to $39.8 \%$ in the control group, with highest values in the Social Functioning Scale for child selfand parent proxy-reports from the patient and comparison samples. We also observed greater ceiling (18.0-
40.6\%) than floor effects $(0.8-5.6 \%)$ in the Cancer Module, with a notable ceiling effect in the Pain and hurt scale and a moderate one in other scales for child selfand parent proxy-reports (Table 3).

\section{Feasibility}

The majority of patients and their parents needed only 10-18 and 6-10 minutes to complete the two questionnaires, respectively. For child self- and parent proxyreport on the PedsQL 4.0 Generic Core Scales, the percentage of missing item responses was $0.6 \%$ and $0.4 \%$, respectively, for all scales except the School Functioning Scale. The percentage of missing items for the School Functioning scale was $21.5 \%$ for child self-report (aged 5-18 years) and $46.3 \%$ for parent proxy-report (aged 218 years). This large percentage missing items for the School Functioning scale may exist because many Chinese children younger than 7 years neither attend kindergarten nor school. For child self- and parent proxyreport on the PedsQL 3.0 Cancer Module, the percentage of missing item responses was $0.9 \%$ and $1.3 \%$, respectively, for all scales. On this module, $40.7 \%$ of the items across all forms had no missing responses. The highest percentage of missing responses for any single item on the Caner Module was 3.2\% for Child selfreport in the Communication II scale (it is had for me to ask the doctors and nurses questions) and 5.9\% for parent proxy-report in the Worryl scale (worry about side effect from medical treatment).

\section{Internal consistency and test-retest reliability}

Internal consistency reliability coefficients are presented in Table 4. Cronbach's alpha for Total Scale of the Generic Scales and the Caner Scale were all above 0.7 in both self- and proxy-reports. Most scales exceeded the minimum reliability standard of 0.70 , and a number of scales approached or met the reliability criterion of 0.90 recommended for analyzing individual patient scores.

Retests for reliability were completed by 27 children with cancer (aged 5-18 years) and 34 patents of such children (aged 2-18 years) who completed the initial questionnaires. ICCs for test-retest reliability for child self- and parent proxy-report are presented in Table 4. All of these ICCs are in the good to excellent reliability range.

\section{Validity}

Table 2 demonstrates the differences between healthy children and children with cancer. For each Generic Core Scale, children with cancer and their parents report statistically significant lower HRQOL than healthy children. Table 5 provides the result comparing the three groups of patients in known distinct clinical conditions (on-treatment, off treatment $\leq 12$ months 
Table 1 Characteristics of the patients with cancer and healthy children

\begin{tabular}{|c|c|c|c|c|c|c|c|c|c|c|}
\hline \multirow[t]{3}{*}{ Variables } & \multicolumn{10}{|c|}{ Patients with cancer } \\
\hline & \multicolumn{2}{|c|}{$\begin{array}{l}\text { Child On-treatment } \\
(n=157)\end{array}$} & \multicolumn{2}{|c|}{$\begin{array}{l}\text { Child off-treatment }< \\
12 \mathrm{~m}(\mathrm{n}=35)\end{array}$} & \multicolumn{2}{|c|}{$\begin{array}{l}\text { Child off-treatment > } \\
12 \mathrm{~m}(\mathrm{n}=74)\end{array}$} & \multicolumn{2}{|c|}{$\begin{array}{l}\text { Total sample } \\
(\mathrm{n}=266)\end{array}$} & \multicolumn{2}{|c|}{$\begin{array}{l}\text { Healthy Children } \\
(\mathrm{n}=284)\end{array}$} \\
\hline & $\mathrm{n}$ & $\%$ & $\mathrm{n}$ & $\%$ & $\mathrm{n}$ & $\%$ & $\mathrm{n}$ & $\%$ & $\mathrm{n}$ & $\%$ \\
\hline \multicolumn{11}{|l|}{ Age } \\
\hline $2-4$ & 47 & 29.9 & 9 & 25.7 & 8 & 10.8 & 64 & 24.1 & 68 & 23.9 \\
\hline $5-7$ & 42 & 26.8 & 10 & 28.6 & 25 & 33.8 & 77 & 28.9 & 81 & 28.5 \\
\hline $8-12$ & 38 & 24.2 & 8 & 22.9 & 23 & 31.1 & 69 & 25.9 & 74 & 26.1 \\
\hline 13-18 & 30 & 19.1 & 8 & 22.9 & 18 & 24.3 & 56 & 21.1 & 61 & 21.5 \\
\hline \multicolumn{11}{|l|}{ Gender† } \\
\hline Male & 92 & 58.6 & 20 & 57.1 & 42 & 56.8 & 154 & 57.9 & 159 & 56.0 \\
\hline Female & 65 & 41.4 & 15 & 42.9 & 32 & 43.2 & 112 & 42.1 & 125 & 44.0 \\
\hline \multicolumn{11}{|l|}{ Diagnosis } \\
\hline Leukemia or lymphoma & 91 & 58.0 & 22 & 62.9 & 50 & 67.6 & 163 & 61.3 & - & \\
\hline Solid tumor & 66 & 42.0 & 13 & 37.1 & 24 & 32.4 & 103 & 38.7 & - & \\
\hline \multicolumn{11}{|l|}{ Education } \\
\hline Non-attendance & 50 & 31.8 & 9 & 25.7 & 12 & 16.2 & 71 & 26.7 & 0 & 0.0 \\
\hline Current school attendance & 107 & 68.2 & 26 & 74.3 & 62 & 83.8 & 195 & 73.3 & 284 & 100.0 \\
\hline \multicolumn{11}{|c|}{ Parents } \\
\hline Mother & 131 & 83.4 & 27 & 77.1 & 62 & 83.8 & 220 & 82.7 & 237 & 83.5 \\
\hline Father & 26 & 16.6 & 8 & 22.9 & 12 & 16.2 & 46 & 17.3 & 47 & 16.5 \\
\hline
\end{tabular}

$\mathrm{n}$ : number of individuals.

and off-treatment $>12$ months) for child self- and parent proxy-report on the PedsQL Generic Core Scales and Cancer Module. For both child self- and parent proxy-report, the PedsQL 4.0 Generic Core Scale Total Score, Physical Functioning, and Emotional Functioning scores demonstrated significant differences between the patients on-treatment and off-treatment $>12$ months. For the PedsQL 3.0 Caner Module Scales, children who had been off-treatment over 12 months and their parents demonstrated significant higher scores than children who had been on-treatment on the Pain and hurt, Nausea, and Procedural Anxiety subscales. In addition,

Table 2 Scale descriptives for PedsQL 4.0 Generic Core Scales child self- and parent proxy-report, and comparisons between children with cancer and healthy children scores

\begin{tabular}{|c|c|c|c|c|c|c|c|c|}
\hline \multirow[t]{2}{*}{ Scale } & \multicolumn{3}{|c|}{ Children with cancer } & \multicolumn{3}{|c|}{ Healthy children } & \multirow[t]{2}{*}{ Effect size* } & \multirow[t]{2}{*}{$t+$} \\
\hline & $\mathrm{n}$ & Mean (SD) & \%Floor/\%Ceiling & $\mathrm{n}$ & Mean (SD) & \%Floor/\%Ceiling & & \\
\hline \multicolumn{9}{|l|}{ Child Self-report } \\
\hline Total score & 202 & $68.56(17.4)$ & $0.00 / 4.0$ & 216 & $84.72(16.4)$ & $0.00 / 6.5$ & 0.96 & 9.77 \\
\hline Physical functioning & 202 & $67.96(19.9)$ & $0.00 / 11.4$ & 216 & $86.33(16.1)$ & $0.00 / 18.5$ & 1.03 & 10.39 \\
\hline Psychosocial functioning & 202 & $69.20(18.4)$ & $0.00 / 5.1$ & 216 & $84.20(17.3)$ & $0.00 / 16.2$ & 0.86 & 8.59 \\
\hline Emotional functioning & 202 & $68.40(20.9)$ & $0.00 / 12.9$ & 215 & $80.25(15.8)$ & $0.00 / 37.2$ & 0.68 & 6.57 \\
\hline Social functioning & 202 & $78.31(16.1)$ & $0.00 / 19.8$ & 216 & $88.16(17.6)$ & $0.00 / 39.8$ & 0.57 & 5.95 \\
\hline School functioning & 165 & $62.19(22.5)$ & $0.00 / 8.5$ & 213 & $83.81(16.2)$ & $0.00 / 31.9$ & 1.15 & 10.87 \\
\hline \multicolumn{9}{|l|}{ Parent Proxy-report } \\
\hline Total score & 266 & $65.54(18.8)$ & $0.00 / 2.6$ & 284 & $80.74(16.9)$ & $0.00 / 5.6$ & 0.87 & 9.81 \\
\hline Physical functioning & 266 & $66.13(20.5)$ & $0.38 / 10.9$ & 284 & $82.18(17.6)$ & $0.00 / 15.8$ & 0.92 & 9.88 \\
\hline Psychosocial functioning & 266 & $65.06(18.7)$ & $0.00 / 4.2$ & 284 & $80.09(17.6)$ & $0.00 / 15.1$ & 0.84 & 9.69 \\
\hline Emotional functioning & 266 & $64.07(19.5)$ & $0.00 / 11.3$ & 284 & $76.90(16.6)$ & $0.00 / 33.8$ & 0.73 & 8.32 \\
\hline Social functioning & 266 & $73.49(17.6)$ & $0.00 / 18.8$ & 284 & $85.33(15.4)$ & $0.00 / 38.0$ & 0.70 & 8.42 \\
\hline School functioning & 195 & $60.55(23.6)$ & $0.00 / 6.7$ & 284 & $78.03(16.5)$ & $0.00 / 29.9$ & 0.87 & 9.54 \\
\hline
\end{tabular}

SD: standard deviation.

*Effect size for differences in means are designated as small (0.20-0.49), medium (0.50-0.79), and large (0.80 and above) in magnitude.

†All scale scores for the children with caner are significant differences from healthy children $(p<.0 .01)$ based on the independent sample $t$ test. 
Table 3 Scale descriptives of the PedsQL 3.0 Cancer Module for child self- and parent proxy-reports

\begin{tabular}{lllllll}
\hline Scale & \multicolumn{3}{c}{ Child self-reports } & \multicolumn{3}{c}{ Parent proxy-reports } \\
& $\mathbf{n}$ & Mean (SD) & \%Floor/\%Ceiling & $\mathbf{n}$ & Mean (SD) & \%Floor/\%Ceiling \\
\hline Pain and hurt & 202 & $74.24(21.89)$ & $1.0 / 40.6$ & 266 & $77.41(20.72)$ & $0.8 / 38.0$ \\
Nausea & 202 & $75.73(20.54)$ & $1.5 / 37.1$ & 266 & $73.21(19.45)$ & $1.9 / 33.8$ \\
Procedural anxiety & 202 & $68.02(27.34)$ & $4.5 / 28.2$ & 266 & $64.14(24.73)$ & $5.6 / 20.8$ \\
Treatment anxiety & 202 & $71.68(24.24)$ & $4.0 / 26.7$ & 266 & $67.13(23.69)$ & $3.0 / 20.1$ \\
Worry & 201 & $68.14(25.19)$ & $3.0 / 31.6$ & 262 & $62.92(24.30)$ & $1.9 / 23.2$ \\
Cognitive problems & 200 & $71.72(29.33)$ & $3.0 / 18.5$ & 260 & $73.93(26.60)$ & $1.2 / 18.0$ \\
Perceived physical appearance & 202 & $76.35(20.47)$ & $3.5 / 33.8$ & 261 & $77.91(17.21)$ & $0.8 / 28.0$ \\
Communication & 201 & $72.19(20.19)$ & $1.5 / 24.7$ & 262 & $71.63(23.61)$ & $1.5 / 25.0$ \\
\hline
\end{tabular}

Table 4 Reliability of the Chinese mandarin version of the PedsQL 4.0 Generic Core Scales and 3.0 Cancer Module: self- and proxy-reports

\begin{tabular}{|c|c|c|c|c|c|c|c|c|c|c|c|}
\hline \multirow[t]{3}{*}{ Scale } & \multicolumn{10}{|c|}{ Internal consistency reliability } & \multirow{3}{*}{$\begin{array}{l}\text { Retest reliability } \\
\text { ICC }\end{array}$} \\
\hline & \multicolumn{2}{|c|}{$2-4$ years } & \multicolumn{2}{|c|}{$5-7$ years } & \multicolumn{2}{|c|}{ 8-12 years } & \multicolumn{2}{|c|}{ 13-18 years } & \multicolumn{2}{|l|}{ Total } & \\
\hline & $\mathrm{n}$ & $\alpha$ & n & $\alpha$ & $\mathrm{n}$ & $\alpha$ & $\mathrm{n}$ & $\alpha$ & $\mathrm{n}$ & $\alpha$ & \\
\hline \multicolumn{12}{|l|}{ Generic Scale } \\
\hline \multirow[t]{2}{*}{ Total score } & NA & & 77 & 0.82 & 69 & 0.85 & 56 & 0.89 & 202 & 0.86 & 0.81 \\
\hline & 64 & 0.87 & 77 & 0.90 & 69 & 0.92 & 56 & 0.91 & 266 & 0.91 & 0.83 \\
\hline \multirow[t]{2}{*}{ Physical functioning } & NA & & 77 & 0.65 & 69 & 0.83 & 56 & 0.82 & 202 & 0.81 & 0.90 \\
\hline & 64 & 0.84 & 77 & 0.88 & 69 & 0.85 & 56 & 0.86 & 266 & 0.84 & 0.89 \\
\hline \multirow[t]{2}{*}{ Psychosocial functioning } & NA & & 77 & 0.72 & 69 & 0.84 & 56 & 0.81 & 202 & 0.82 & 0.79 \\
\hline & 64 & 0.85 & 77 & 0.87 & 69 & 0.88 & 56 & 0.87 & 266 & 0.86 & 0.78 \\
\hline \multirow[t]{2}{*}{ Emotional functioning } & NA & & 77 & 0.68 & 69 & 0.78 & 56 & 0.76 & 202 & 0.73 & 0.74 \\
\hline & 64 & 0.72 & 77 & 0.73 & 69 & 0.76 & 56 & 0.80 & 266 & 0.79 & 0.77 \\
\hline \multirow[t]{2}{*}{ Social functioning } & NA & & 77 & 0.56 & 69 & 0.68 & 56 & 0.79 & 202 & 0.71 & 0.71 \\
\hline & 64 & 0.76 & 77 & 0.82 & 69 & 0.79 & 56 & 0.83 & 266 & 0.80 & 0.75 \\
\hline \multirow[t]{2}{*}{ School functioning } & NA & & 49 & 0.47 & 64 & 0.66 & 55 & 0.69 & 165 & 0.65 & 0.78 \\
\hline & 27 & 0.68 & 49 & 0.75 & 64 & 0.72 & 55 & 0.71 & 195 & 0.72 & 0.84 \\
\hline \multicolumn{12}{|l|}{ Cancer scale } \\
\hline \multirow[t]{2}{*}{ Total scale } & NA & & 77 & 0.70 & 69 & 0.79 & 56 & 0.81 & 202 & 0.77 & 0.76 \\
\hline & 64 & 0.83 & 77 & 0.85 & 69 & 0.87 & 56 & 0.88 & 266 & 0.87 & 0.84 \\
\hline \multirow[t]{2}{*}{ Pain and hurt } & NA & & 77 & 0.66 & 69 & 0.70 & 56 & 0.72 & 202 & 0.69 & 0.61 \\
\hline & 64 & 0.80 & 77 & 0.83 & 69 & 0.87 & 56 & 0.90 & 266 & 0.88 & 0.75 \\
\hline \multirow[t]{2}{*}{ Nausea } & NA & & 77 & 0.78 & 69 & 0.80 & 56 & 0.85 & 202 & 0.81 & 0.63 \\
\hline & 64 & 0.83 & 77 & 0.83 & 69 & 0.88 & 56 & 0.86 & 266 & 0.85 & 0.79 \\
\hline \multirow[t]{2}{*}{ Procedural anxiety } & NA & & 77 & 0.74 & 69 & 0.82 & 56 & 0.84 & 202 & 0.76 & 0.77 \\
\hline & 64 & 0.85 & 77 & 0.86 & 69 & 0.89 & 56 & 0.88 & 266 & 0.89 & 0.73 \\
\hline \multirow[t]{2}{*}{ Treatment anxiety } & NA & & 77 & 0.75 & 59 & 0.81 & 56 & 0.83 & 202 & 0.78 & 0.75 \\
\hline & 64 & 0.88 & 77 & 0.87 & 59 & 0.87 & 56 & 0.89 & 266 & 0.88 & 0.61 \\
\hline \multirow[t]{2}{*}{ Worry } & NA & & 76 & 0.55 & 59 & 0.76 & 56 & 0.81 & 201 & 0.75 & 0.82 \\
\hline & 61 & 0.90 & 76 & 0.86 & 59 & 0.85 & 56 & 0.88 & 262 & 0.87 & 0.79 \\
\hline \multirow[t]{2}{*}{ Cognitive problems } & NA & & 75 & 0.50 & 59 & 0.70 & 56 & 0.74 & 200 & 0.74 & 0.68 \\
\hline & 59 & 0.71 & 76 & 0.79 & 59 & 0.78 & 56 & 0.87 & 260 & 0.80 & 0.80 \\
\hline \multirow[t]{2}{*}{ Perceived physical appearance } & NA & & 77 & 0.54 & 59 & 0.65 & 56 & 0.71 & 201 & 0.61 & 0.74 \\
\hline & 60 & 0.62 & 76 & 0.71 & 59 & 0.74 & 56 & 0.80 & 261 & 0.75 & 0.80 \\
\hline \multirow[t]{2}{*}{ Communication } & NA & & 76 & 0.62 & 59 & 0.70 & 56 & 0.73 & 202 & 0.69 & 0.72 \\
\hline & 60 & 0.87 & 77 & 0.82 & 59 & 0.84 & 56 & 0.81 & 262 & 0.84 & 0.70 \\
\hline
\end{tabular}

Reliability of parent proxy-report is set in boldface.

NA: not applicable; $\alpha$ : Cronbach's coefficient alpha; ICC: intraclass correlation coefficient. 
Table 5 One-way ANOVA comparing HRQOL (generic scale and cancer scale) between children on- and off-treatment ( $\leq 12$ months or > 12 months): self- and proxy-report (bold)

\begin{tabular}{|c|c|c|c|c|c|c|c|c|c|}
\hline \multirow[t]{2}{*}{ Scales } & \multicolumn{2}{|c|}{ Child On-treatment (a) } & \multicolumn{2}{|c|}{$\begin{array}{l}\text { Child off-treatment (b) } \\
\leq 12 \text { months }\end{array}$} & \multicolumn{2}{|c|}{$\begin{array}{l}\text { Child off-treatment (c) } \\
\quad>12 \text { months }\end{array}$} & \multirow[t]{2}{*}{ Difference } & \multirow[t]{2}{*}{$\mathrm{F}$} & \multirow[t]{2}{*}{$\begin{array}{c}P \\
\text { Value }\end{array}$} \\
\hline & $\mathbf{n}$ & mean (SD) & $\mathrm{n}$ & Mean (SD) & $n$ & Mean (SD) & & & \\
\hline \multicolumn{10}{|l|}{ Generic Scale } \\
\hline \multirow[t]{2}{*}{ Total score } & 110 & $65.42(16.98)$ & 26 & $67.14(18.05)$ & 66 & 73.69 (16.19) & $a<c^{* *}$ & 5.04 & 0.007 \\
\hline & 157 & $62.33(16.70)$ & 35 & $65.92(19.43)$ & 74 & $71.22(17.92)$ & $\mathrm{a}<\mathrm{c}^{* * *}$ & 6.58 & 0.002 \\
\hline \multirow[t]{2}{*}{ Physical functioning } & 110 & $62.57(21.08)$ & 26 & $67.12(18.25)$ & 66 & 76.26 (19.24) & $a<c^{* * *}, b<c^{*}$ & 9.53 & 0.000 \\
\hline & 157 & $61.55(18.67)$ & 35 & $66.18(20.63)$ & 74 & $75.51(21.15)$ & $\mathrm{a}<\mathrm{c}^{* * *}, \mathrm{~b}<\mathrm{c}^{*}$ & 12.7 & 0.000 \\
\hline \multirow[t]{2}{*}{ Psychosocial functioning } & 110 & $67.94(17.53)$ & 26 & $67.23(19.77)$ & 66 & 72.20 (18.92) & & 1.30 & 0.275 \\
\hline & 157 & $63.07(19.40)$ & 35 & $66.04(17.36)$ & 74 & $68.87(18.56)$ & & 2.42 & 0.091 \\
\hline \multirow[t]{2}{*}{ Emotional functioning } & 110 & $64.71(19.54)$ & 26 & $67.36(22.81)$ & 66 & 73.64 (20.16) & $a<c^{* *}$ & 4.05 & 0.019 \\
\hline & 157 & $59.82(24.58)$ & 35 & $68.40(21.82)$ & 74 & 70.71 (18.44) & $a<b^{*}, a<c^{* * *}$ & 6.44 & 0.002 \\
\hline \multirow[t]{2}{*}{ Social functioning } & 110 & 77.97 (17.48) & 26 & 75.03 (18.24) & 66 & 79.30 (15.68) & & 0.59 & 0.556 \\
\hline & 157 & $72.02(20.68)$ & 35 & $72.26(18.52)$ & 74 & 75.16 (18.79) & & 0.65 & 0.524 \\
\hline \multirow[t]{2}{*}{ School functioning } & 86 & 58.47 (19.89) & 20 & 60.35 (18.55) & 59 & 65.18 (23.09) & & 1.81 & 0.167 \\
\hline & 107 & $57.96(19.30)$ & 26 & $60.80(22.25)$ & 62 & $62.94(24.35)$ & & 1.08 & 0.342 \\
\hline \multicolumn{10}{|l|}{ Cancer scale } \\
\hline \multirow[t]{2}{*}{ Pain and hurt } & 110 & 70.23 (20.54) & 26 & 75.35 (20.19) & 66 & 78.66 (22.47) & $a<c^{*}$ & 3.38 & 0.036 \\
\hline & 157 & $74.68(19.03)$ & 35 & $77.81(21.45)$ & 74 & $82.12(20.60)$ & $a<c^{* *}$ & 3.57 & 0.029 \\
\hline \multirow[t]{2}{*}{ Nausea } & 110 & $63.92(22.36)$ & 26 & 78.72 (19.11) & 66 & 83.20 (18.59) & $a<b^{* *}, a<c^{* * *}$ & 19.22 & 0.000 \\
\hline & 157 & $66.81(18.29)$ & 35 & $72.34(23.05)$ & 74 & $81.72(16.14)$ & $a<c^{* * *}, b<c^{*}$ & 16.5 & 0.000 \\
\hline \multirow[t]{2}{*}{ Procedural anxiety } & 110 & $63.17(28.31)$ & 26 & $70.29(25.25)$ & 66 & 73.33 (24.90) & $a<c^{*}$ & 3.12 & 0.046 \\
\hline & 157 & $62.42(22.63)$ & 35 & $64.71(25.39)$ & 74 & $76.26(24.87)$ & $a<c^{* * *}, b<c^{*}$ & 8.74 & 0.000 \\
\hline \multirow[t]{2}{*}{ Treatment anxiety } & 110 & $68.77(23.48)$ & 26 & $72.11(26.23)$ & 66 & $73.84(20.64)$ & & 1.05 & 0.352 \\
\hline & 157 & $62.85(20.69)$ & 35 & $68.05(24.38)$ & 74 & $72.97(23.38)$ & $\mathrm{a}<\mathrm{c}^{* *}$ & 5.46 & 0.005 \\
\hline \multirow[t]{2}{*}{ Worry } & 110 & $66.59(23.24)$ & 26 & $70.33(28.10)$ & 66 & $71.32(25.61)$ & & 0.83 & 0.438 \\
\hline & 155 & $59.13(22.00)$ & 35 & $60.78(24.43)$ & 72 & $67.20(24.74)$ & $a<c^{*}$ & 3.06 & 0.049 \\
\hline \multirow[t]{2}{*}{ Cognitive problems } & 108 & 70.09 (25.65) & 26 & $73.83(30.02)$ & 66 & $71.75(29.45)$ & & 0.22 & 0.804 \\
\hline & 154 & $72.11(23.96)$ & 34 & $74.44(29.12)$ & 72 & $74.25(26.92)$ & & 0.23 & 0.791 \\
\hline \multirow[t]{2}{*}{ Perceived physical appearance } & 110 & 74.85 (19.48) & 26 & 76.98 (22.17) & 66 & 79.65 (18.34) & & 1.24 & 0.291 \\
\hline & 154 & $77.21(16.90)$ & 34 & $74.93(20.55)$ & 73 & $75.77(17.49)$ & & 0.32 & 0.723 \\
\hline \multirow[t]{2}{*}{ Communication } & 110 & 72.66 (19.11) & 26 & 73.27 (18.40) & 65 & 70.98 (22.75) & & 0.18 & 0.832 \\
\hline & 155 & $71.18(22.83)$ & 35 & $75.29(24.52)$ & 72 & 72.04 (22.38) & & 0.46 & 0.633 \\
\hline
\end{tabular}

${ }^{*} \mathrm{P}<0.05$, ${ }^{* * P}<0.01,{ }^{* * * P}<0.001$ based on Tukey Honestly Significantly Different post hoc analysis.

the scores of parent proxy-report Treatment Anxiety and Worry subscale were significant differences between children on-treatment versus off-treatment $>12$ months.

The result of the factor analysis for child self- and parent proxy-report of the Generic Core Scales and Cancer Module are presented in Table 6 and 7. For the Generic Core Scales, an eigenvalue cutoff of 1.0 resulted in a six factor solution for child self- and parent proxy-report, accounting for $62.3 \%$ and $69.6 \%$ of the variance, respectively. For the Cancer Module, an eight factor solution for child selfreport was result, accounting for $78.0 \%$ of the variance; and a seven-factor solution for parent proxy-report was result, accounting for $86.6 \%$ of the variance.

As to the intercorrelations among the various Generic Core Scales and the Cancer Module scales estimated using correlation coefficients. As anticipated, correlation coefficients between the Generic Core Scale Total Scale and the Cancer Module subscales were of medium to large effect size for both the child self- and parent proxy-reports (Table 8 ).

Table 8 presents the correlation between scores of the child self- and parent proxy-reports of the Genetic Core Scales and the Caner Module. A positive correlation between child self- and parent proxy-reports was found on all scales of both the Generic Core Scales and the Cancer Module.

\section{Discussion}

This study demonstrated the feasibility, reliability, and validity of the Chinese mandarin version of PedsQL 4.0 Generic Core Scales and 3.0 Cancer Module in children with cancer living in mainland China. 
Table 6 PedsQL 4.0 Generic Core Scales factor loadings for child self- and parent proxy-report in children with cancer

\begin{tabular}{|c|c|c|c|c|c|c|c|}
\hline Subscale & Item & Factor 1 & Factor 2 & Factor 3 & Factor 4 & Factor 5 & Factor 6 \\
\hline \multirow[t]{16}{*}{ Physical functioning } & P1 & .666 & .000 & .153 & .065 & .131 & .126 \\
\hline & & .338 & .700 & .078 & .041 & .064 & .005 \\
\hline & P2 & .825 & -.037 & .127 & .101 & -.021 & .123 \\
\hline & & -.031 & .779 & .250 & .092 & .121 & .243 \\
\hline & P3 & .828 & .047 & -.029 & -.135 & .100 & -.022 \\
\hline & & -.020 & .756 & -.093 & .168 & .025 & .042 \\
\hline & P4 & .804 & .274 & -.052 & -.249 & .004 & .036 \\
\hline & & -.123 & .807 & -.116 & .157 & .210 & .062 \\
\hline & P5 & .721 & .468 & .091 & .241 & -.024 & -.034 \\
\hline & & .185 & .693 & .153 & -.046 & .190 & .151 \\
\hline & P6 & .373 & -.002 & .203 & .477 & .094 & .051 \\
\hline & & -.043 & .658 & .292 & .082 & .256 & .074 \\
\hline & P7 & .620 & .139 & -.103 & .172 & .023 & .072 \\
\hline & & .620 & .345 & .055 & -.189 & .028 & .186 \\
\hline & P8 & .527 & .228 & .059 & .121 & .114 & -.157 \\
\hline & & .571 & .073 & .108 & .183 & .259 & .068 \\
\hline \multirow[t]{10}{*}{ Emotional functioning } & E1 & -.018 & .070 & .830 & .165 & .082 & .130 \\
\hline & & -.049 & .225 & -.129 & .048 & .777 & .009 \\
\hline & E2 & .026 & .188 & .710 & .026 & .102 & .035 \\
\hline & & .093 & .225 & -.021 & .179 & .780 & -.163 \\
\hline & E3 & .136 & .217 & .688 & .105 & .135 & .121 \\
\hline & & .225 & .182 & .012 & .007 & .748 & .134 \\
\hline & E4 & -.005 & .002 & .263 & -.013 & .071 & .755 \\
\hline & & .205 & .148 & .050 & .726 & .377 & -.070 \\
\hline & E5 & .173 & -.206 & .305 & -.048 & -.084 & .638 \\
\hline & & .200 & .143 & -.082 & .555 & .407 & -.268 \\
\hline \multirow[t]{10}{*}{ Social functioning } & S1 & .227 & .697 & .209 & .179 & -.302 & .199 \\
\hline & & .202 & .152 & .700 & .293 & -.158 & -.128 \\
\hline & S2 & .160 & .848 & .005 & .009 & .069 & .178 \\
\hline & & -.026 & -.017 & .367 & .777 & .058 & .124 \\
\hline & S3 & -.025 & .695 & .217 & .126 & .216 & -.271 \\
\hline & & -.155 & .040 & .563 & .217 & .232 & .176 \\
\hline & S4 & .199 & .412 & .781 & .256 & -.064 & .009 \\
\hline & & -.203 & .039 & .506 & .089 & .268 & .074 \\
\hline & S5 & .025 & .668 & .265 & .318 & .198 & .131 \\
\hline & & .017 & .254 & .756 & .092 & .044 & .003 \\
\hline \multirow[t]{10}{*}{ School functioning } & Sc1 & -.207 & .378 & .006 & .716 & -.059 & .069 \\
\hline & & .161 & .078 & .134 & -.102 & .138 & .664 \\
\hline & Sc2 & -.038 & .031 & .255 & .802 & .021 & -.170 \\
\hline & & -.040 & -.014 & .187 & .189 & .039 & .521 \\
\hline & $\mathrm{Sc} 3$ & -.068 & .187 & .089 & .600 & .194 & .072 \\
\hline & & .179 & .041 & .181 & .052 & .047 & .833 \\
\hline & Sc4 & .154 & .096 & .021 & .040 & .923 & -.094 \\
\hline & & .860 & .135 & -.045 & .181 & -.012 & .290 \\
\hline & Sc5 & .030 & .044 & .253 & .095 & .908 & .152 \\
\hline & & .111 & .233 & .015 & -.119 & .110 & .813 \\
\hline \multirow[t]{2}{*}{ Eigenvalue } & & 6.744 & 2.844 & 2.208 & 1.878 & 1.579 & 1.145 \\
\hline & & 7.457 & 3.554 & 2.453 & 1.956 & 1.699 & 1.182 \\
\hline \multirow[t]{2}{*}{ Percent Variance } & & 25.32 & 10.364 & 9.602 & 7.164 & 5.865 & 3.977 \\
\hline & & 28.074 & 11.103 & 10.665 & 8.071 & 6.517 & 5.138 \\
\hline
\end{tabular}

Note: Bold values indicate the largest factor loading for each item.

In each cell, child self-report loading are shown above and the parent proxy-report loading are shown below in italics.

Extraction Method: Principle Component Analysis. Rotation Method: Oblimin with Kaiser Normalization. Total Variance Explained for child self-report: 62.3\%; for

Parent Proxy-report: 69.6\%. 
Table 7 PedsQL 3.0 Cancer Module factor loadings for child self- and parent proxy-report in children with cancer

\begin{tabular}{|c|c|c|c|c|c|c|c|c|c|}
\hline Subscale & Item & Factor 1 & Factor 2 & Factor 3 & Factor 4 & Factor 5 & Factor 6 & Factor 7 & Factor 8 \\
\hline \multirow[t]{4}{*}{ Pain and hurt } & P1 & .034 & .104 & .008 & .023 & .689 & .150 & .071 & .104 \\
\hline & & -.009 & .023 & .029 & -.16 & .066 & .009 & .533 & \\
\hline & P2 & .136 & .206 & .072 & .049 & .714 & -.171 & .031 & .073 \\
\hline & & .097 & .075 & .017 & .180 & .091 & .056 & .789 & \\
\hline \multirow[t]{10}{*}{ Nausea } & N1 & .854 & .115 & .121 & .039 & -.06 & -.173 & -.123 & .219 \\
\hline & & .158 & .835 & -.057 & .087 & -.086 & -.079 & .016 & \\
\hline & N2 & .657 & .028 & -.094 & .044 & .253 & .005 & .007 & .130 \\
\hline & & .004 & .573 & .080 & -.038 & .021 & .022 & -.046 & \\
\hline & N3 & .789 & .018 & .082 & -.009 & .107 & -.094 & -.02 & -.007 \\
\hline & & .199 & .827 & -.013 & .156 & -.057 & .069 & .134 & \\
\hline & N4 & .668 & -.183 & .038 & .019 & .032 & .111 & -.04 & -.072 \\
\hline & & .052 & .731 & -.123 & .070 & .075 & .147 & .343 & \\
\hline & N5 & .821 & -.008 & .145 & .114 & .049 & .138 & -.077 & -.033 \\
\hline & & .190 & .727 & .089 & .026 & .244 & .080 & .093 & \\
\hline \multirow[t]{6}{*}{ Procedural anxiety } & PA1 & .011 & .159 & .042 & .866 & .061 & .103 & -.133 & .199 \\
\hline & & .766 & -.114 & .043 & -.061 & .070 & -.047 & .184 & \\
\hline & PA2 & .050 & .043 & -.03 & .876 & .055 & -.100 & .035 & -.08 \\
\hline & & .860 & .156 & .030 & -.022 & -.156 & .070 & .361 & \\
\hline & PA3 & -.057 & .137 & .212 & .917 & .011 & -.069 & .099 & .061 \\
\hline & & .848 & .035 & -.044 & -.095 & -.007 & .062 & .005 & \\
\hline \multirow[t]{6}{*}{ Treatment anxiety } & TA1 & .350 & .840 & .040 & .158 & .135 & .009 & .133 & -.1 \\
\hline & & .821 & .024 & .085 & .248 & .114 & .105 & .045 & \\
\hline & TA2 & .071 & .795 & .127 & .030 & .135 & .069 & .193 & -.068 \\
\hline & & .864 & .290 & .092 & .096 & .210 & .157 & -.082 & \\
\hline & TA3 & .156 & .861 & .011 & .186 & .293 & -.101 & -.021 & -.035 \\
\hline & & .888 & .155 & .116 & .245 & .046 & .041 & -.099 & \\
\hline \multirow[t]{6}{*}{ Worry } & W1 & .049 & .013 & .622 & -.011 & .127 & .028 & .049 & -.095 \\
\hline & & .033 & .043 & .118 & .561 & .040 & .060 & .063 & \\
\hline & W2 & .253 & .172 & .751 & .176 & .033 & .044 & .083 & .003 \\
\hline & & .021 & .024 & .014 & .775 & .142 & -.022 & .191 & \\
\hline & W3 & .066 & .021 & .807 & .125 & .004 & .058 & .062 & .201 \\
\hline & & -.054 & .099 & .078 & .775 & .020 & .184 & .004 & \\
\hline \multirow[t]{10}{*}{ Cognitive problems } & CP1 & -.197 & -.03 & .012 & .120 & .031 & .882 & .105 & .038 \\
\hline & & .196 & .137 & .849 & -.051 & .273 & .087 & .163 & \\
\hline & $\mathrm{CP} 2$ & .004 & -.165 & -.04 & -.034 & -.083 & .863 & .079 & .138 \\
\hline & & .112 & .051 & .9 & .034 & .070 & .219 & .073 & \\
\hline & CP3 & -.137 & -.041 & -.198 & .011 & .391 & .631 & .120 & -.009 \\
\hline & & -.013 & -.004 & .614 & .191 & .243 & .091 & .172 & \\
\hline & $\mathrm{CP} 4$ & -.002 & .221 & .006 & -.119 & .085 & .706 & .098 & .109 \\
\hline & & .223 & -.044 & .742 & .002 & .180 & .279 & -.057 & \\
\hline & CP5 & -.204 & .134 & -.109 & -.073 & .177 & .665 & .074 & -.022 \\
\hline & & .061 & .085 & .794 & .155 & .176 & -.053 & -.16 & \\
\hline \multirow[t]{6}{*}{ Perceived physical appearance } & A1 & .005 & .820 & -.14 & .050 & -.087 & -.024 & -.192 & .076 \\
\hline & & .041 & .161 & .246 & .088 & .112 & .767 & .270 & \\
\hline & $\mathrm{A} 2$ & .024 & .757 & .046 & .050 & -.215 & -.073 & -.094 & .013 \\
\hline & & .060 & -.094 & -.162 & .179 & .212 & .637 & -.062 & \\
\hline & $\mathrm{A} 3$ & .213 & .857 & .166 & -.198 & -.18 & .036 & .033 & -.047 \\
\hline & & -.079 & -.106 & .030 & .142 & .079 & .666 & -.03 & \\
\hline \multirow[t]{3}{*}{ Communication } & $\mathrm{C} 1$ & .123 & .101 & .182 & .118 & .159 & .020 & .060 & .611 \\
\hline & & .009 & .131 & .074 & .044 & .843 & .311 & -.016 & \\
\hline & C2 & -.083 & -.107 & .078 & .040 & .148 & .1 & -.041 & .905 \\
\hline
\end{tabular}


Table 7 PedsQL 3.0 Cancer Module factor loadings for child self- and parent proxy-report in children with cancer (Continued)

\begin{tabular}{|c|c|c|c|c|c|c|c|c|c|}
\hline & \multirow{3}{*}{ C3 } & .024 & .181 & .193 & .175 & .551 & -.148 & .046 & \\
\hline & & .010 & -.005 & .281 & .098 & .048 & .035 & -.188 & .742 \\
\hline & & .113 & -.144 & .110 & .040 & .743 & .105 & .061 & \\
\hline \multirow[t]{2}{*}{ Eigenvalue } & & 6.548 & 4.303 & 3.635 & 2.319 & 2.074 & 1.771 & 1.34 & 1.096 \\
\hline & & 8.682 & 5.225 & 3.725 & 2.756 & 2.142 & 1.843 & 1.447 & \\
\hline \multirow[t]{2}{*}{ Percent Variance } & & 24.253 & 13.936 & 9.462 & 7.587 & 7.182 & 6.56 & 4.962 & 4.057 \\
\hline & & 32.157 & 15.647 & 1.091 & 8.613 & 8.081 & 6.715 & 5.246 & \\
\hline
\end{tabular}

Note: Bold values indicate the largest factor loading for each item.

In each cell, child self-report loading are shown above and the parent proxy-report loading are shown below in italics.

Extraction Method: Principle Component Analysis. Rotation Method: Oblimin with Kaiser Normalization. Total Variance Explained for child self-report: 78.0\%; for Parent Proxy-report: $86.6 \%$

With regard to obtention of result, only a short amount of time was required to complete the questionnaires. This short completion time made these two instruments particularly applicable to the fast-pace setting of an outpatient clinic. Additionally, the overall percentage of missing item responses across the PedsQL scales was low, indicating that children and their parents were able to provide good data regarding the child's HRQOL. However, our results showed that there were several items, i.e. 'worry about side effect from medical treatment' and 'it is had for me to ask the doctors and nurses questions', had a high missing rate. We found that the percentage of missing values was primarily from the 2-7 years old children. The reason may be that some parents regarded their children as too young to understand the questions, or some children aged 5-7 years had difficult to understand these questions, since not all of them attend kindergarten or school. This finding is comparable with previous reports of the PedsQL Cancer Module [9], and also in yline with other PedsQL disease-specific modules [38]. This indicated that some modifications for the items of these subscales in Toddler and Young Child version scale were necessary.

No (for Generic Core Scales) or minimal (for the Cancer Module) floor effects and more accentuated ceiling effects for both scales means that distinction by Chinese

Table 8 Intercorrelations among PedsQL Scales and correlation between scores of the child and parent

\begin{tabular}{|c|c|c|c|c|c|c|c|c|c|c|c|c|c|c|}
\hline & Tot & $\mathrm{PH}$ & Psy & Em & Soc & Sch & $\mathbf{P}$ & $\mathrm{N}$ & PA & TA & W & $\mathrm{CP}$ & A & $C$ \\
\hline Total Score (Tot) & $\underline{0.493^{* *}}$ & $0.826^{* *}$ & $0.853^{* *}$ & $0.818^{* *}$ & $0.744^{* *}$ & $0.620^{* *}$ & $0.476^{* *}$ & $0.428^{* *}$ & $0.434^{* *}$ & $0.380^{* *}$ & $0.395^{* *}$ & $0.336^{* *}$ & $0.304^{* *}$ & $0.318^{* *}$ \\
\hline $\begin{array}{l}\text { Physical } \\
\text { functioning (Ph) }\end{array}$ & $0.878^{* *}$ & $\underline{0.501^{* *}}$ & $0.591^{* *}$ & $0.506^{* *}$ & $0.530^{* *}$ & $0.486^{* *}$ & $0.429^{* *}$ & $0.278^{* *}$ & $0.316^{* *}$ & $0.280^{* *}$ & $0.303^{* *}$ & $0.245^{* *}$ & $0.266^{* *}$ & $0.271^{* *}$ \\
\hline $\begin{array}{l}\text { Psychosocial } \\
\text { functioning (Psy) }\end{array}$ & $0.905^{* *}$ & $0.585^{* *}$ & $\underline{0.336^{* *}}$ & $0.647^{* *}$ & $0.631^{* *}$ & $0.677^{* *}$ & $0.298^{* *}$ & $0.322^{* *}$ & $0.303^{* *}$ & $0.254^{* *}$ & $0.370^{* *}$ & $0.333^{* *}$ & $0.395^{* *}$ & $0.257^{* *}$ \\
\hline $\begin{array}{l}\text { Emotional } \\
\text { functioning (Em) }\end{array}$ & $0.860^{* *}$ & $0.494^{* *}$ & $0.848^{* *}$ & $\underline{0.327^{* *}}$ & $0.507^{* *}$ & $0.293^{* *}$ & $0.231^{* *}$ & $0.210^{* *}$ & $0.287^{* *}$ & $0.224^{* *}$ & $0.266^{* *}$ & $0.205^{* *}$ & $0.239^{* *}$ & $0.194^{*}$ \\
\hline $\begin{array}{l}\text { Social } \\
\text { functioning (Soc) }\end{array}$ & $0.798^{* *}$ & $0.535^{* *}$ & $0.869^{* *}$ & $0.605^{* *}$ & $\underline{0.359^{*}}$ & $0.413^{* *}$ & $0.203^{* *}$ & 0.136 & $0.206^{* *}$ & $0.264^{* *}$ & $0.277^{* *}$ & $0.248^{* *}$ & $0.233^{* *}$ & $0.220^{* *}$ \\
\hline $\begin{array}{l}\text { School } \\
\text { functioning (Sch) }\end{array}$ & $0.729^{* *}$ & $0.497^{* *}$ & $0.785^{* *}$ & $0.375^{* *}$ & $0.523^{* *}$ & $\underline{0.305^{* *}}$ & $0.356^{* *}$ & & $0.274^{* *}$ & $0.297^{* *}$ & $0.283^{* *}$ & $0.394^{* *}$ & $0.195^{* *}$ & 0.118 \\
\hline Pain and hurt (P) & $0.513^{* *}$ & $0.447^{* *}$ & $0.461^{* *}$ & $0.413^{* *}$ & $0.386^{* *}$ & $0.342^{* *}$ & $\underline{0.442^{* *}}$ & $0.228^{* *}$ & $0.297^{* *}$ & $0.273^{* *}$ & $0.465^{* *}$ & $0.305^{* *}$ & $0.341^{* *}$ & $0.282^{* *}$ \\
\hline Nausea (N) & $0.479 * *$ & $0.302^{* *}$ & $0.337^{* *}$ & $0.314^{* *}$ & $0.291^{* *}$ & $0.292^{* *}$ & $0.220^{* *}$ & $\underline{0.406^{* *}}$ & $0.235^{*}$ & $0.365^{* *}$ & $0.353^{* *}$ & $0.238^{*}$ & $0.174^{*}$ & 0.120 \\
\hline $\begin{array}{l}\text { Procedural } \\
\text { anxiety (PA) }\end{array}$ & $0.365^{* *}$ & $0.278^{* *}$ & $0.285^{* *}$ & $0.290^{* *}$ & $0.272^{* *}$ & $0.228^{* *}$ & $0252^{* *}$ & 0.032 & $\underline{0.349 * *}$ & $0.483^{* *}$ & $0.377^{* *}$ & $0.174^{*}$ & $0.319^{* *}$ & $0.230^{* *}$ \\
\hline $\begin{array}{l}\text { Treatment } \\
\text { anxiety (TA) }\end{array}$ & $0.326^{* *}$ & $0.298^{* *}$ & $0.382^{* *}$ & $0.324^{* *}$ & $0.273^{* *}$ & $0.204^{* *}$ & $0.189^{*}$ & $0.239^{* *}$ & $0.434^{* *}$ & $\underline{0.420^{*}}$ & $0.329^{* *}$ & $0.341^{* *}$ & $0.260^{* *}$ & $0.285^{* *}$ \\
\hline Worry (W) & $0.396^{* *}$ & $0.237^{* *}$ & $0.410^{* *}$ & $0.228^{* *}$ & $0.239 * *$ & $0.210^{* *}$ & $0.281^{* *}$ & $0.281^{*}$ & $0.203^{* *}$ & $0.371^{* *}$ & $\underline{0.354^{* *}}$ & $0.214^{* *}$ & $0.357^{* *}$ & $0.219^{* *}$ \\
\hline $\begin{array}{l}\text { Cognitive } \\
\text { problems (P) }\end{array}$ & $0.348^{* *}$ & $0.365^{* *}$ & $0.376^{* *}$ & $0.253^{* *}$ & $0.327^{* *}$ & $0.424^{* *}$ & 0.086 & 0.139 & $0.251^{* *}$ & $0.349^{* *}$ & $0.311^{* *}$ & $\underline{0.308^{*}}$ & $0.398^{* *}$ & $0.276^{* *}$ \\
\hline Appearance (A) & $0.315^{* *}$ & $0.249^{* *}$ & $0.329^{* *}$ & $0.295^{* *}$ & $0.285^{* *}$ & $0.219^{* *}$ & $0.253^{* *}$ & $0.182^{*}$ & $0.184^{*}$ & $0.335^{* *}$ & $0.274^{* *}$ & $0.388^{* *}$ & $\underline{0.322^{* *}}$ & $0.199 * *$ \\
\hline $\begin{array}{l}\text { Communication } \\
\text { (C) }\end{array}$ & $0.354^{* *}$ & $0.213^{* *}$ & $0.311^{* *}$ & $0.291^{* *}$ & $0.271^{* *}$ & $0.231^{* *}$ & $0.270^{* *}$ & 0.118 & $0.321^{* *}$ & $0.293^{* *}$ & 0.042 & $0.251^{* *}$ & $\overline{0.267^{* *}}$ & $\underline{0.318^{* *}}$ \\
\hline
\end{tabular}


mandarin version of the PedsQL 4.0 Generic Core Scale and 3.0 Cancer Module between children who do extremely well or just well is less than excellent [39]. On the other hand, this finding support the opinion that the PedsQL scaling range is acceptable for use in patients experiencing greater health-related problems, which is the area of most concern in research with severe or chronic illness [40,41].

For internal consistency, both the PedsQL 4.0 Generic Core Scales and 3.0 Cancer Module reliabilities approached or exceeded the alpha coefficient standard of 0.7 for most scales. The PedsQL 4.0 Generic Core Scales Total Score for parent proxy-report exceeded an alpha of 0.90 , recommended for individual patient analysis, making the Total Scale score suitable as a summary score for the primary analysis of HRQOL outcome in clinical trials and other group comparisons [42]. The total score of the School Functioning, Pain and hurt, Perceived physical appearance, and Communication subscales for child self-report did not approach or exceed 0.70 . These findings are consistent with reliability estimates seen in the original English version and the German Version $[9,19]$. This low internal consistency may be related to the small number of items that compose the subscales and the low level of schooling in the sample. Although Cronbach's alpha represents the lower bound of the reliability of a measurement instrument, and is a conservative estimate of actual reliability [43], scale that did not meet the 0.70 standard should be used only for descriptive analyses.

It is recommended that the interval between measurements must be long enough to reduce the effects of memory and short enough to diminish the likelihood of systemic alterations. Previous studies found that a period of 1 to 4 weeks is considered adequate [44,45]. In this study, we used a 1-3 weeks interval. Meanwhile, patients were selected who were considered to be stable and were not expected to change before completing the questionnaires for the second time. All scales for both the child self- and parent proxy-reports showed good to excellent reliability, indicating that the Chinese mandarin version of PedsQL 4.0 Generic Core Scale and Cancer Module are stable over time.

As can be expected, the PedsQL 4.0 Generic Core Scales indicated better HRQOL in children of the general population than in children with caner on all scales, which support the construct validity of the translated instrument. Additionally, we found that in child selfand parent proxy-report, physical health subscale scores and some psychosocial health subscale scores in children off-treatment over 12 months were significantly higher than children on-treatment in the two instruments. This result was similar to another study assessing HRQOL scores between children on-treatment and those who were off-treatment [33]. Meanwhile, we found that many psychosocial health subscale scores were not significant improved among children who had been treatment over 12 months. This finding is also in line with studies of HRQOL in children with cancer $[19,20,33]$. It would seem to reflect the long-term burden of psychosocial trials which individuals face as they grow older: they may be afraid, often on an unconscious level, that the disease will recur, they may experience a setback resulting from the stresses during treatment or they can be confronted with new problems evolving from the illness or long-term side effects of treatment [46]. We, like others, believe that psychosocial support remains important long after treatment has completed, and even when the physical health appears well [21,47].

The results of the factor analysis in general support the hypothesized factor structure of the PedsQL. For the PedsQL 4.0 Generic Core Scales, all items split into two different factors. The results do not resemble Varni's five factor structure in the original PedSQL version [41]. In their study, Emotional Functioning items in both child self- and parent reports do not split into two different factors. But the findings of factor analysis may be sample-specific, other studies showed different results too $[27,48]$, this is why the factor structure should be reinvestigated in clinical samples. For the PedsQL Cancer Module, exploratory factor analysis identified 8 factors for child self-reports, replicating those of the original theoretical dimensions. In parent proxy-reports, those items of the Procedural anxiety and Treatment anxiety subscales of the original theoretical dimension loaded on the same factor, reducing the number of factors to 7. In mainland China, parents generally believe that the fear of injection, surgery or other invasive treatments is the main reason why their children try to avoid going to the hospital [49], so that their worry about treatment may linked to the 'Procedural anxiety' factor.

Consistent with the conceptualization of disease-specific symptoms as causal indicators of generic HRQOL, the intercorrelations between the PedsQL 4.0 Generic Core Scales total score and PedsQL 3.0 Cancer Module were in medium to large range, supporting construct validity. Regarding the agreement between child selfand parent proxy-reports, our data showed moderate to good agreement both for the Generic Core Scales and the Cancer Module. Finding higher correlations for the observable parameters in general, like the Physical Functioning Scale in the Generic Core Scale and Pain and hurt, Nausea, and Treatment anxiety in the Cancer Module. This finding is consistent with that of previous studies [33,38]. Although child self-reports is critical, perspectives of parents also are important. In clinical practice, there may be circumstances when the child is too young, or too ill too complete an 
instrument, and parent proxy-report may be needed in such cases. Additionally, it is typically parents' perceptions of their children's HRQOL and symptoms that influences health care utilization. Therefore, in cases in which pediatric patients are not able to provide selfreport, reliable and valid parent proxy-report instrument are needed [50].

This study has several inherent limitations. One limitation of the study was that the study sample was entirely composed of patients seeking medical evaluation or treatment for cancer and cannot be considered representative of the general population of pediatric oncology patients. In mainland China, many children with cancer have refused or abandoned treatment for financial reasons, which is unknown in developed countries and regions [51]. Information about the HRQOL in these patients is still unknown. Second, all the subjects in this study were recruited in two of the largest cities (Shanghai and Chongqing) in China. The majority of our patients came from urban areas instead of rural areas. In fact, more than half of China's population now lives in rural areas where only a few children under 14 years of age who have acute leukemia receive protocol based therapy [23]. Therefore, further studies conducted in rural areas are suggested. Third, information on participants' socioeconomic status was not available. Questions still exist as to whether socioeconomic status is associated with HRQOL in children with cancer in our society [52].

On the other hand, the information from standardized questionnaires provided a wealth of information about the physical and psychosocial status of children with cancer living in mainland China. Moreover, we compared the patients with normal children matched for sex and age, and all subjects were recruited from the same health care catchment areas and assessment were carried out in closely related time periods in both groups. Therefore, the study of these children could provide a more accurate picture of pediatric cancer patients referring to clinical practices, and provide relevant clues for future interventions that promote care and support of children with cancer.

\section{Conclusion}

In summary, our results generally support the feasibility, reliability and validity of the Chinese mandarin version of PedsQL 4.0 Generic Core Scales and the Cancer Module. Further studies should focus on testing responsiveness of the Chinese mandarin version scales in longitudinal studies and in other areas, particularly in rural areas. Studies measuring HRQOL in children who have refused or abandoned treatment are also warranted.

\section{Acknowledgements}

This work was supported by the Department of Pediatric Surgery, Children's Hospital of Fudan University. Dr Varni holds the copyright for the PedsQL. No institution was involved in the analysis of interpretation of data, in writing the article, or in the decision to submit the paper for publication. The authors are indebted to all participating practitioners and doctors' assistant for their participation in the study. The authors are also indebted to all reviewers for their kindly reviewing of the manuscript.

\section{Author details}

${ }^{1}$ Department of Pediatric Surgery, Children's Hospital of Fudan University, Shanghai, 201102, China. 'Department of Pediatric Healthcare, Children's Hospital of Fudan University, Shanghai, 201102, China. ${ }^{3}$ Department of rehabilitation center, Children's Hospital of Chongqing Medical University, Chongqing, 400014, China.

\section{Authors' contributions}

YJ contributed to the design of the study, the conception and interpretation of the statistical analysis, and drafted the manuscript. SYC conducted the statistical analysis, contributed to the interpretation of data, the drafting of the manuscript. $\mathrm{KL}, \mathrm{NX}, \mathrm{XY}$ contributed the acquisition of data. SZ and XMX contributed to the conception and design of the study, and revised of the manuscript. All authors read and approved the final manuscript.

\section{Competing interests}

The authors declare that they have no competing interests.

Received: 30 August 2011 Accepted: 23 November 2011

Published: 23 November 2011

\section{References}

1. Collins JJ, Byrnes ME, Dunkel IJ, Lapin J, Nadel T, Thaler HT, Polyak T, Rapkin B, Portenoy RK: The measurement of symptoms in children with cancer. J Pain Symptom Manage 2000, 19(5):363-377.

2. Erickson JM, Beck SL, Christian BR, Dudley W, Hollen PJ, Albritton KA, Sennett M, Dillon RL, Godder K: Fatigue, sleep-wake disturbances, and quality of life in adolescents receiving chemotherapy. J Pediatr Hematol Oncol 2011, 33(1):e17-e25.

3. Weintraub N, Rot I, Shoshani N, Pe'Er J, Weintraub M: Participation in daily activities and quality of life in survivors of retinoblastoma. Pediatr Blood Cancer 2011, 56(4):590-594.

4. Gurney JG, Tersak JM, Ness KK, Landier W, Matthay KK, Schmidt ML: Hearing loss, quality of life, and academic problems in long-term neuroblastoma survivors: a report from the Children's Oncology Group. Pediatrics 2007, 120(5):e1229-e1236.

5. Barr RD, Chalmers D, De Pauw S, Furlong W, Weitzman S, Feeny D: Healthrelated quality of life in survivors of Wilms' tumor and advanced neuroblastoma: aA cross-sectional study. J Clin Oncol 2000, 18(18):3280-3287.

6. Nathan PC, Ness KK, Greenberg ML, Hudson M, Wolden S, Davidoff A, Laverdiere C, Mertens A, Whitton J, Robison LL, et al: Health-related quality of life in adult survivors of childhood Wilms tumor or neuroblastoma: A report from the childhood cancer survivor study. Pediatr Blood Cancer 2007, 49(5):704-715.

7. Grad FP: The Preamble of the Constitution of the World Health Organization. Bull World Health Organ 2002, 80(12):981-984.

8. Testa MA, Simonson DC: Assesment of quality-of-life outcomes. N Engl J Med 1996, 334(13):835-840.

9. Felder-Puig R, Frey E, Proksch K, Varni JW, Gadner H, Topf R: Validation of the German version of the Pediatric Quality of Life Inventory (PedsQL) in childhood cancer patients off treatment and children with epilepsy. Qual Life Res 2004, 13(1):223-234.

10. Matza LS, Swensen AR, Flood EM, Secnik K, Leidy NK: Assessment of health-related quality of life in children: a review of conceptual, methodological, and regulatory issues. Value Health 2004, 7(1):79-92.

11. Varni JW, Katz ER, Colegrove RJ, Dolgin M: Adjustment of children with newly diagnosed cancer: cross-informant variance. J Psychosoc Oncol 1995, 13(3):23-38.

12. Varni JW, Seid M, Rode CA: The PedsQL: measurement model for the pediatric quality of life inventory. Med Care 1999, 37(2):126-139. 
13. Klaassen RJ, Krahn M, Gaboury I, Hughes J, Anderson R, Grundy P, Ali SK, Jardine $L$, Abla $O$, Silva $M$, et al: Evaluating the ability to detect change of health-related quality of life in children with Hodgkin disease. Cancer 2010, 116(6):1608-1614

14. Marino BS, Shera D, Wernovsky G, Tomlinson RS, Aguirre A, Gallagher M, Lee A, Cho CJ, Stern W, Davis L, et al: The development of the pediatric cardiac quality of life inventory: a quality of life measure for children and adolescents with heart disease. Qual Life Res 2008, 17(4):613-626.

15. Palmer SN, Meeske KA, Katz ER, Burwinkle TM, Varni JW: The PedsQL Brain Tumor Module: initial reliability and validity. Pediatr Blood Cancer 2007, 49(3):287-293.

16. Varni JW, Burwinkle TM, Berrin SJ, Sherman SA, Artavia K, Malcarne VL, Chambers HG: The PedsQL in pediatric cerebral palsy: reliability, validity, and sensitivity of the Generic Core Scales and Cerebral Palsy Module. Dev Med Child Neurol 2006, 48(6):442-449.

17. Varni JW, Burwinkle TM, Jacobs JR, Gottschalk M, Kaufman F, Jones KL: The PedsQL in type 1 and type 2 diabetes: reliability and validity of the Pediatric Quality of Life Inventory Generic Core Scales and type 1 Diabetes Module. Diabetes Care 2003, 26(3):631-637.

18. Varni JW, Burwinkle TM, Rapoff MA, Kamps $J$, Olson N: The PedsQL in pediatric asthma: reliability and validity of the Pediatric Quality of Life Inventory generic core scales and asthma module. J Behav Med 2004, 27(3):297-318

19. Varni JW, Burwinkle TM, Katz ER, Meeske K, Dickinson P: The PedsQL in pediatric cancer: reliability and validity of the Pediatric Quality of Life Inventory Generic Core Scales, Multidimensional Fatigue Scale, and Cancer Module. Cancer 2002, 94(7):2090-2106.

20. Tsuji N, Kakee N, Ishida Y, Asami K, Tabuchi K, Nakadate H, Iwai T, Maeda M, Okamura J, Kazama T, et al: Validation of the Japanese version of the Pediatric Quality of Life Inventory (PedsQL) Cancer Module. Health Qual Life Outcomes 2011, 9:22.

21. Sitaresmi MN, Mostert S, Gundy CM, Sutaryo Veerman AJ: Health-related quality of life assessment in Indonesian childhood acute lymphoblastic leukemia. Health Qual Life Outcomes 2008, 6:96.

22. Scarpelli AC, Paiva SM, Pordeus IA, Ramos-Jorge ML, Varni JW, Allison PJ: Measurement properties of the Brazilian version of the Pediatric Quality of Life Inventory (PedsQL) cancer module scale. Health Qual Life Outcomes 2008, 6:7.

23. Ribeiro RC, Pui CH: Saving the children-improving childhood cancer treatment in developing countries. N Engl J Med 2005, 352(21):2158-2160.

24. Varni JW, Katz ER, Seid M, Quiggins DJ, Friedman-Bender A, Castro CM: The Pediatric Cancer Quality of Life Inventory (PCQL). I. Instrument development, descriptive statistics, and cross-informant variance. J Behav Med 1998, 21(2):179-204.

25. Varni JW, Katz ER, Seid M, Quiggins DJ, Friedman-Bender A: The pediatric cancer quality of life inventory-32 (PCQL-32): I. Reliability and validity. Cancer 1998, 82(6):1184-1196.

26. Varni JW, Seid M, Rode CA: The PedsQL: measurement model for the pediatric quality of life inventory. Med Care 1999, 37(2):126-139.

27. Yang $X$, Xiao N, Yan J: The PedsQL in pediatric cerebral palsy: reliability and validity of the Chinese version pediatric quality of life inventory 4.0 generic core scales and 3.0 cerebral palsy module. Qual Life Res 2011, 20(2):243-252.

28. Hao Y, Tian Q, Lu Y, Chai Y, Rao S: Psychometric properties of the Chinese version of the Pediatric Quality of Life Inventory 4.0 generic core scales. Qual Life Res 2010, 19(8):1229-1233.

29. McHorney CA, Ware JJ, Lu JF, Sherbourne CD: The MOS 36-item ShortForm Health Survey (SF-36): III. Tests of data quality, scaling assumptions, and reliability across diverse patient groups. Med Care 1994, 32(1):40-66.

30. Cronbach $\sqcup$ : Coefficient alpha and the internal structure of test. Psychometrika 1951, 16(1):297-334.

31. Wilson KA, Dowling AJ, Abdolell M, Tannock IF: Perception of quality of life by patients, partners and treating physicians. Qual Life Res 2000, 9(9):1041-1052.

32. Cohen J: In Statistical Power Analysis for the Behavioral Sciences. Volume 2. New Jersey: Lawrence Erlbaum Associates; 1988.

33. Scarpelli AC, Paiva SM, Pordeus IA, Ramos-Jorge ML, Varni JW, Allison PJ: Measurement properties of the Brazilian version of the Pediatric Quality of Life Inventory (PedsQL) cancer module scale. Health Qual Life Outcomes 2008, 6:7.
34. Tsuji N, Kakee N, Ishida Y, Asami K, Tabuchi K, Nakadate H, Iwai T, Maeda M, Okamura J, Kazama T, et al: Validation of the Japanese version of the Pediatric Quality of Life Inventory (PedsQL) Cancer Module. Health Qual Life Outcomes 2011, 9:22.

35. Fayers PM, Hand DJ: Factor analysis, causal indicators and quality of life. Qual Life Res 1997, 6(2):139-150.

36. Floyd FJ, Widaman KF: Factor analysis in the development and refinement of clinical assessment instruments. Psychological Assess 1995, 7(3):286-299.

37. Bartko JJ: The intraclass correlation coefficient as a measure of reliability. Psychol Rep 1966, 19(1):3-11.

38. Varni JW, Seid M, Smith KT, Burwinkle T, Brown J, Szer IS: The PedsQL in pediatric rheumatology: reliability, validity, and responsiveness of the Pediatric Quality of Life Inventory Generic Core Scales and Rheumatology Module. Arthritis Rheum 2002, 46(3):714-725.

39. Piko BF: Self-perceived health among adolescents: the role of gender and psychosocial factors. Eur J Pediatr 2007, 166(7):701-708.

40. Bindman $A B$, Keane $D$, Lurie $N$ : Measuring health changes among severely ill patients. The floor phenomenon. Med Care 1990, 28(12):1142-1152.

41. Varni JW, Seid M, Kurtin PS: PedsQL 4.0: reliability and validity of the Pediatric Quality of Life Inventory version 4.0 generic core scales in healthy and patient populations. Med Care 2001, 39(8):800-812.

42. Nunnally J, Bernstein I: In Psychometric theory. Volume 3. New York: McGrawHill; 1994.

43. Novick MR, Lewis C: Coefficient alpha and the reliability of composite measurements. Psychometrika 1967, 32(1):1-13.

44. Marino BS, Tomlinson RS, Wernovsky G, Drotar D, Newburger JW, Mahony L, Mussatto K, Tong E, Cohen M, Andersen C, et al: Validation of the pediatric cardiac quality of life inventory. Pediatrics 2010, 126(3):498-508.

45. Jenkinson C: Evaluating the efficacy of medical treatment: possibilities and limitations. Soc Sci Med 1995, 41(10):1395-1401.

46. Mulhern RK, Wasserman AL, Friedman AG, Fairclough D: Social competence and behavioral adjustment of children who are long-term survivors of cancer. Pediatrics 1989, 83(1):18-25.

47. Krull KR, Huang S, Gurney JG, Klosky JL, Leisenring W, Termuhlen A, Ness KK, Kumar SD, Mertens A, Stovall M, et al: Adolescent behavior and adult health status in childhood cancer survivors. J Cancer Surviv 2010, 4(3):210-217

48. Reinfjell T, Diseth TH, Veenstra M, Vikan A: Measuring health-related quality of life in young adolescents: reliability and validity in the Norwegian version of the Pediatric Quality of Life Inventory 4.0 (PedsQL) generic core scales. Health Qual Life Outcomes 2006, 4:61.

49. Yu WY, Yang GF, Ren ZM: [Survey of psychological health of parents of children with malignant tumor]. Di Yi Jun Yi Da Xue Xue Bao 2004, 24(10):1197-1198.

50. Tomlinson D, Hinds PS, Bartels U, Hendershot E, Sung L: Parent reports of quality of life for pediatric patients with cancer with no realistic chance of cure. J Clin Oncol 2011, 29(6):639-645.

51. Tang $Y, X u X$, Song $H$, Yang S, Shi S, Wei J: Long-term outcome of childhood acute lymphoblastic leukemia treated in China. Pediatr Blood Cancer 2008, 51(3):380-386.

52. Mostert S, Sitaresmi MN, Gundy CM, Sutaryo Veerman AJ: Influence of socioeconomic status on childhood acute lymphoblastic leukemia treatment in Indonesia. Pediatrics 2006, 118(6):e1600-e1606.

doi:10.1186/1477-7525-9-103

Cite this article as: Ji et al:: Measuring health-related quality of life in children with cancer living in mainland China: feasibility, reliability and validity of the Chinese mandarin version of PedsQL 4.0 Generic Core Scales and 3.0 Cancer Module. Health and Quality of Life Outcomes 2011 9:103. 